\title{
Fatores de risco associados à colonização por Candida spp em neonatos internados em uma Unidade de Terapia Intensiva Neonatal brasileira
}

\author{
Risk factors associated with colonization by Candida spp in neonates \\ hospitalized in a Neonatal Intensive Care Unit in Brazil
}

\author{
Raniery Martins Borges ${ }^{1}$, Leandro Rafael Soares ${ }^{1}$, Cristiane Silveira de Brito ${ }^{1}$, \\ Denise Von Dolinger de Brito ${ }^{1}$, Vânia Olivetti Steffen Abdallah ${ }^{2}$ e Paulo Pinto Gontijo Filho ${ }^{1}$
}

\begin{abstract}
RESUMO
Os objetivos desse estudo foram investigar a participação de Candida albicans e não-albicans como agente de colonização e sepse, bem como os fatores de risco associados aos neonatos internados na Unidade de Terapia Intensiva Neonatal do Hospital de Clínicas da Universidade Federal de Uberlândia. Foi realizada vigilância epidemiológica pelo sistema National Healthcare Safety Network no período entre agosto de 2007 e abril de 2008. A taxa de incidência de sepse com critério microbiológico foi de 6,7/1.000 paciente/dia, constatando-se apenas um caso de candidemia. Aproximadamente, $19 \%$ dos neonatos estavam colonizados por Candida, identificadas como Candida albicans (50\%) e Candida não-albicans (50\%). Os fatores de risco significantes para colonização por Candida spp foram a idade gestacional entre 26 e 30 semanas, o uso prévio de antibiótico e o cateter vascular central umbilical. A mortalidade total foi de $11,8 \%$ nos neonatos internados durante o período de estudo com sepse, porém 0 recém-nascido com candidemia não evoluiu para óbito.
\end{abstract}

Palavras-chaves: Neonatos críticos. Colonização. Candida spp. Fator de risco.

\begin{abstract}
The objectives of this study were to investigate the participation of Candida albicans and non-albicans as colonization and sepsis agents, along with the risk factors associated with the neonates in the neonatal intensive care unit of the clinical hospital of the Federal University of Uberlândia. Epidemiological surveillance was implemented through the National Healthcare Safety Network between August 2007 and April 2008. The incidence rate for sepsis with microbiological criteria was 6.7/1,000 patients/day, which was shown as only one case of candidemia. Approximately $19 \%$ of the neonates were colonized by Candida, which was identified as Candida albicans (50\%) and Candida not-albicans (50\%). The significant risk factors for Candida spp colonization were gestational age of between 26 and 30 weeks, previous antibiotic use and umbilical central vascular catheter. The overall mortality among the neonates hospitalized with sepsis over the study period was $11.8 \%$. However, the neonate with candidemia did not die.
\end{abstract}

Key-words: Critical neonates. Colonization. Candida spp. Risk factor.

A sepse fúngica está tornando-se cada vez mais freqüente no período neonatal, especialmente nos neonatos de muito baixo peso, sendo usualmente uma condição grave que acomete preferencialmente os recém-nascidos submetidos a procedimentos invasivos e, está relacionada à elevada morbi-mortalidade ${ }^{12}$.

\footnotetext{
1. Laboratório de Microbiologia, Instituto de Ciências Biomédicas, Universidade Federal de Uberlândia, Uberlândia, MG. 2. Hospital de Clínicas, Universidade Federal de Uberlândia, Uberlândia, MG.

Endereço para Correspondência: Dra. Denise Von Dolinger de Brito. Laboratório de Microbiologia/UFU. Campus Umuarama. Av. Amazonas s/n, Bloco 4C/2 $2^{\circ}$ andar, 38400-000 Uberlândia, MG.

Tel: 5534 3218-2236

e-mail: denisebrito@terra.com.br

Recebido para publicação em 23/04/2009

Aceito em 11/08/2009
}

Candida albicans é a principal espécie capaz de provocar infecção hospitalar em Unidade de Terapia Intensiva Neonatal (UTIN), entretanto, outras espécies não-albicans tiveram aumento em suas frequências nos últimos anos incluindo a Candida tropicalis, Candida parapsilosis, Candida krusei e Candida glabrata ${ }^{19}$.

Acredita-se que a maioria dos episódios de candidemia sejam adquiridos de origem endógena, pela translocação do patógeno através da mucosa do trato gastrointestinal, local onde a colonização por este fungo ocorre em até $70 \%$ da população normal $^{13}$. Qualquer variável que provoque desequilíbrio da microbiota ou lesão da mucosa gastrointestinal pode ser um agente facilitador de translocação de Candida spp do lúmen intestinal para os capilares mesentéricos ${ }^{3}$.

Além da prematuridade, inúmeros fatores de risco contribuem para infecção e colonização por Candida, tais como: extremo e 
muito baixo peso, estrutura imatura da pele, idade gestacional, tempo de internação, o uso prolongado de antimicrobianos, inserção de cateter venoso central, nutrição parenteral, ventilação mecânica, uso de esteróides e colonização fúngica preexistente ${ }^{141518}$.

Os objetivos desse estudo foram investigar a participação de Candida albicans e não-albicans como agente de colonização e sepse, bem como os fatores de risco associados à colonização em neonatos internados na UTIN do Hospital de Clínicas da Universidade Federal de Uberlândia (HC-UFU).

\section{MATERIAL E MÉTODOS}

Instituição. 0 Hospital de Clínicas da Universidade Federal de Uberlândia é um hospital de ensino, assistência terciária, com 510 leitos. A UTIN compreende dez leitos e faz parte do Berçário de Alto Risco da instituição.

Vigilância de infecção. Foi realizada vigilância epidemiológica pelo sistema National Healthcare Safety Network (NHSN) para avaliação da ocorrência de candidemias em neonatos críticos, no período agosto de 2007 a abril de 2008. Os neonatos foram monitorados diariamente em busca de infecção de corrente sanguínea por esses microrganismos. Adicionalmente, foram realizadas coletas semanais de mucosas oral e perianal para avaliar a relação entre colonização prévia e infecção por representantes do gênero Candida spp de todos os pacientes internados no período do estudo.

Técnicas microbiológicas. Coleta de materiapesquisa de colonização: foram realizadas coletas semanais de mucosa oral e perianal, com o auxílio de swab estéril préumedecido com salina estéril e com Trypticase Soy Broth (TSB), respectivamente. Os espécimes das cavidades bucal e anal e as amostras provenientes de episódios de candidemia foram cultivadas em placas de CHROMagar ${ }^{\mathrm{TM}}$ Candida (DIFCO- USA) para caracterização presuntiva das colônias em Candida albicans (colônias com coloração verde), Candida tropicalis (colônias com coloração azul), Candida krusei (colônias com coloração rosa) e outras espécies (colônias com coloração branca). As placas foram incubadas à temperatura de $35^{\circ} \mathrm{C}$, por $24 \mathrm{a} 72 \mathrm{hs}$.

Hemoculturas: os espécimes de sangue foram obtidos a partir de punção de veia periférica. As hemoculturas foram realizadas inoculando-se 5 a $10 \mathrm{~mL}$ de sangue em um frasco do sistema comercial automatizado Bactec/Alert ${ }^{\circledR}$ (Vitek System) no laboratório de microbiologia do HC- UFU. Os espécimes positivos foram subcultivados em placa com agar sangue, incubada a $35^{\circ} \mathrm{C}$, por 24 a 48 horas.

Estocagem das amostras. As amostras obtidas de infecção e colonização de mucosas oral e perianal foram estocadas em tubos contendo TSB acrescidos de glicerol $20 \%$ e mantidos no freezer a $-20^{\circ} \mathrm{C}$.

Identificação das espécies de Candida spp. Foram caracterizadas por meio de testes de fermentação dos seguintes carboidratos: glicose, galactose, trealose, maltose, celobiose, rafinose e lactose, utilizando-se o kit de identificação Candifast (I.M. - França, e as leituras foram realizadas após incubação a $35^{\circ} \mathrm{C}$, por $48 \mathrm{hs}$.
Teste de suscetibilidade aos antifúngicos (in vitro). Os inóculos foram obtidos a partir do subcultivo em caldo BHI (Brain Heart Infusion Broth) à temperatura de $35^{\circ} \mathrm{C}$, e incubação por $24 \mathrm{hs}$. A suspensão resultante foi semeada em CHROMagar ${ }^{\mathrm{TM}}$ Candida (DIFCO- USA) e após incubação à $35^{\circ} \mathrm{C}$, foram realizados testes de suscetibilidade aos seguintes antifúngicos: Anfotericina B, Fluconazol, Nistatina, Flucitosina, Econazol, Ketoconazol e Miconazol; utilizando-se o kit Candifast (I.M. - França).

Análise estatística. Foi realizada análise estatística para confirmação da significância dos principais fatores de risco para as infecções. Para isto, foram realizados o teste do $\chi^{2}$ para comparação entre as variáveis qualitativas, o teste exato de Fisher para analisar as variáveis qualitativas com o $\mathbf{n}$ menor ou igual a 5 e o teste t de Student para analisar variáveis quantitativas. Estes dados serão analisados através do programa Epi Info Software versão 2000 (CDC, Atlanta)

Ética do estudo. Esta pesquisa foi aprovada pelo Comitê de Ética em Pesquisa da Universidade Federal de Uberlândia.

\section{RESULTADOS}

No total, 114 neonatos foram incluídos no estudo realizado na UTIN do HC-UFU, no período de agosto de 2007 a abril de 2008, perfazendo um total de 2.532 pacientes/dia. Aproximadamente $40 \%(46 / 114)$ dos pacientes apresentaram sepse clínica e menos da metade (37\%) foram ratificadas por critério microbiológico, incluindo 23,5\% (4/17) com extremo baixo peso $(\leq 1.000 \mathrm{~g})$. A taxa de incidência de sepse com critério microbiológico foi de 6,7/1.000 paciente/dia, constatando-se apenas um caso de candidemia, correspondendo a uma taxa de incidência de 0,4/1.000 paciente/dia. Os principais agentes de sepse foram bactérias do gênero Staphylococcus spp (82,3\%) representados por Staphylococcus coagulase negativa (64,3\%) e o Staphylococcus aureus (35,7\%).

Apesar de ter sido detectado apenas um caso de candidemia $(0,9 \%), 19,3 \%$ (22/114) dos neonatos estavam colonizados por Candida, correspondente a 45 amostras recuperadas de mucosa oral e perianal, sendo caracterizadas como Candida albicans $(51,1 \%$ 23/45), Candida krusei (22,2\% - 10/45), Candida glabrata (15,6\% - 7/45), Candida parapsilosis (8,9\% - 4/45) e Candida tropicalis $(2,2 \%-1 / 45)$ (Figura 1). Esta colonização foi mais freqüiente na região perianal $(68,9 \%)$ do que na boca $(31,1 \%)$.

Os fatores de risco relacionados significativamente à colonização por Candida spp foram: idade gestacional entre $26 \mathrm{e}$ 30 semanas, uso prévio de antibiótico e o uso do cateter vascular central umbilical (Tabela 1).

Das 45 amostras isoladas de neonatos colonizados, 93,3\% (42/45) foram resistentes aos seguintes antifúngicos: anfotericina $\mathrm{B}$, fluconazol, nistatina, flucitosina, econazol, ketoconazol e miconazol, sendo 23 (51,1\%) de Candida albicans, 10 (22,2\%) de Candida krusei, 4 (8,9\%) de Candida parapsilosis, 4 (8,9\%) de Candida glabrata e, 1 (2,2\%) de Candida tropicalis. No total, apenas $3(6,7 \%)$ amostras foram sensíveis aos antifúngicos testados e, corresponderam a Candida glabrata. 


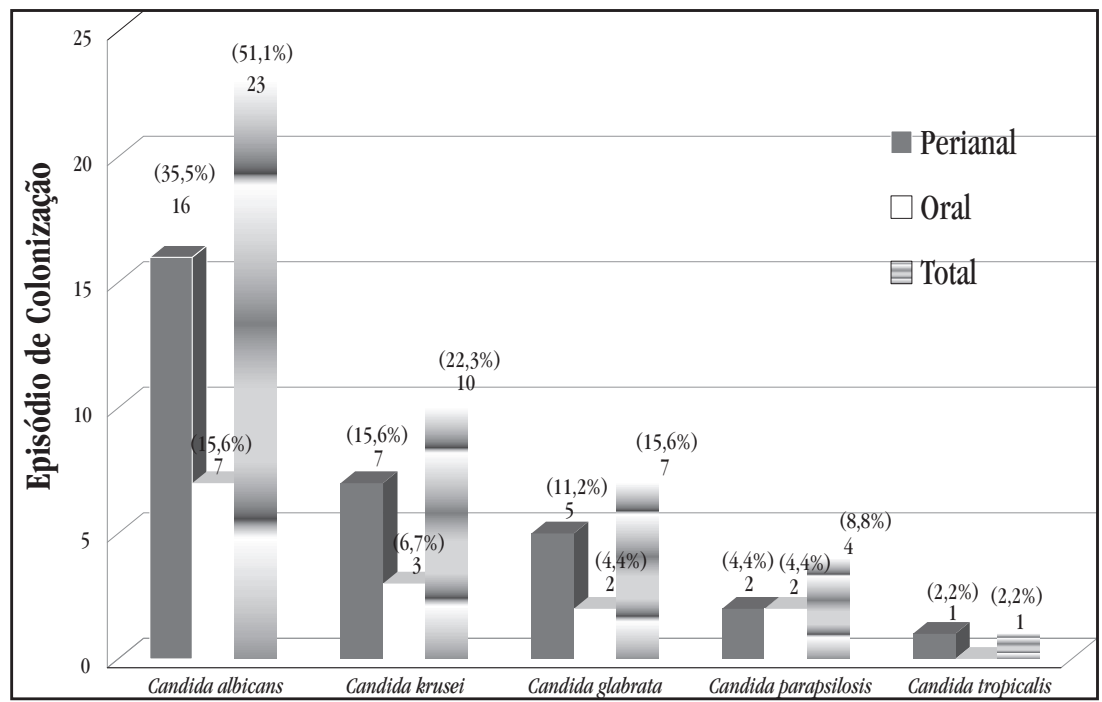

\section{FIGURA 1}

Distribuição dos episódios de colonização por Candida spp e sítio de coleta em neonatos internados no HC-UFU, no período de agosto de 2007 a abril de 2008

\section{TABELA 1}

Fatores de risco para colonização por Candida spp e mortalidade em neonatos internados na Unidade de Terapia Intensiva Neonatal do Hospital de Clínicas da Universidade Federal de Uberlândia no período de agosto de 2007 a abril de 2008.

\begin{tabular}{|c|c|c|c|c|c|c|c|}
\hline \multirow{2}{*}{$\begin{array}{l}\text { Fatores de risco } \\
\text { Peso(g) }\end{array}$} & \multicolumn{2}{|c|}{ Casos } & \multicolumn{2}{|c|}{ Controle } & \multirow[b]{2}{*}{$P$} & \multirow[b]{2}{*}{ OR } & \multirow[b]{2}{*}{$\left(\mathrm{IC}_{03}\right)$} \\
\hline & $\left(n^{0}=22\right)$ & $\%$ & $\left(\mathrm{n}^{\mathrm{0}}=60\right)$ & $\%$ & & & \\
\hline$\leq 1.000$ & 3 & 13,7 & 3 & 5,0 & 0,33 & 3,00 & $(0,43-20,91)$ \\
\hline $1.001-1.500$ & 7 & 31,8 & 22 & 36,7 & 0,88 & 0,81 & $(0,25-2,54)$ \\
\hline $1.505-2.500$ & 5 & 22,7 & 19 & 31,7 & 0,60 & 0,63 & $(0,17-2,21)$ \\
\hline$>2.500$ & 7 & 31,8 & 16 & 26,6 & 0,85 & 1,28 & $(0,39-4,18)$ \\
\hline \multicolumn{8}{|l|}{ Idade gestacional (semanas) } \\
\hline$<26$ & 0 & 0,0 & 2 & 3,3 & 1,00 & 0,00 & $(0,00-11,63)$ \\
\hline $26-30$ & 8 & 36,4 & 8 & 13,3 & $0,02^{*}$ & 3,71 & $(1,03-13,58)$ \\
\hline $31-36$ & 7 & 31,8 & 35 & 60,0 & 0,06 & 0,33 & $(0,10-1,04)$ \\
\hline$\geq 37$ & 7 & 31,8 & 14 & 23,4 & 0,62 & 1,53 & $(0,46-5,10)$ \\
\hline \multicolumn{8}{|c|}{ Tempo de hospitalização (dias) } \\
\hline$\leq 7$ & 7 & 31,8 & 32 & 53,3 & 0,13 & 0,41 & $(0,13-1,27)$ \\
\hline$>8$ & 15 & 68,2 & 28 & 46,7 & 0,13 & 2,45 & $(0,79-7,82)$ \\
\hline \multicolumn{8}{|l|}{ SNAP-II } \\
\hline $0-9$ & 9 & 40,9 & 17 & 28,3 & 0,41 & 1,75 & $(0,56-5,45)$ \\
\hline $10-19$ & 7 & 31,8 & 28 & 46,7 & 0,69 & 0,70 & $(0,21-2,28)$ \\
\hline$\geq 20$ & 6 & 27,3 & 15 & 25,0 & 0,93 & 1,13 & $(0,32-3,83)$ \\
\hline \multicolumn{8}{|l|}{ SNAPPE-II } \\
\hline $0-9$ & 8 & 36,4 & 17 & 28,3 & 0,66 & 1,45 & $(0,45-4,57)$ \\
\hline $10-19$ & 6 & 27,2 & 21 & 35,0 & 0,69 & 0,70 & $(0,21-2,28)$ \\
\hline$\geq 20$ & 8 & 36,4 & 22 & 36,7 & 0,81 & 0,99 & $(0,32-3,04)$ \\
\hline Entubado & 11 & 50,0 & 16 & 26,7 & 0,08 & 2,75 & $(0,89-8,57)$ \\
\hline NPT & 14 & 63,6 & 32 & 53,3 & 0,56 & 1,53 & $(0,50-4,72)$ \\
\hline Uso prévio de antibiótico & 20 & 90,9 & 24 & 40,0 & $<0,01^{*}$ & 15,00 & $(2,94-102,55)$ \\
\hline \multicolumn{8}{|l|}{ Em uso de CVC } \\
\hline intracath & 3 & 15,8 & 1 & 2,6 & 0,09 & 7,13 & $(0,58-192,72)$ \\
\hline flebotomia & 6 & 31,6 & 7 & 17,9 & 0,31 & 2,11 & $(0,50-8,94)$ \\
\hline PICC & 10 & 52,6 & 23 & 59,0 & 0,86 & 0,77 & $(0,22-2,68)$ \\
\hline Umbilical & 0 & 0,0 & 8 & 20,5 & $0,04^{*}$ & 0,00 & $(0,00-1,26)$ \\
\hline Mortalidade & 4 & 18,2 & 3 & 5,0 & 0,07 & 4,22 & $(0,70-26,89)$ \\
\hline
\end{tabular}

$\mathrm{P}=\leq 0,05$, OR: odds ratio, IC: intervalo de confiança, SNAP-II: score for neonatal acute physiology II, SNAPPE-II: score for neonatal acute physioloy perinatal extension, NPT: nutrição parenteral total, CVC: cateter vascular central, PICC: cateter central de inserção periférica 
A mortalidade total foi de $11,8 \%$ nos neonatos internados durante o período de estudo com sepse, mas o recém-nascido com candidemia não evoluiu para óbito.

\section{DISCUSSÃ0}

Neste estudo, aproximadamente 40\% (46/114) dos neonatos apresentaram sepse clínica e 36,9\% (17/46) destes pacientes apresentaram sepse com diagnóstico microbiológico, observandose uma incidência de 6,7/1.000 paciente/dia.

A maioria das infecções fúngicas neonatais é causada por Candida, particularmente Candida albicans. Entretanto, as espécies Candida parapsilosis e Candida tropicalis estão se tornando freqüentes nos últimos anos, inclusive em surtos de UTIN $^{917}$. No período investigado, foi encontrado somente um caso de candidemia por Candida albicans.

A candidemia é causa significante de morbidade e mortalidade em pacientes críticos e imunocomprometidos, e contribui para o aumento no tempo de hospitalização e nos custos médicos ${ }^{5}$. Neste trabalho, houve apenas um caso de candidemia detectado no HCUFU; entretanto, a criança veio transferida de outro hospital.

Sastre e cols ${ }^{16}$, em estudo prospectivo multicêntrico incluindo 20.565 neonatos admitidos em 27 unidades neonatais na Espanha, relataram 6 casos de candidíase sistêmica. Linder e $\operatorname{cols}^{8}$, em Israel, registraram candidemia em 1.3\% de 4.201 recém-nascidos admitidos em uma UTIN. A frequência de candidemia é maior nos estudos que incluem somente recém-nascidos de baixo peso, com variação de 2,2 a 12,9\% nos recém nascidos com peso inferior a $1.500 \mathrm{~g}$ e de $5,5 \%$ a $16,5 \%$ naqueles com menos de $1.000 \mathrm{~g}^{18}$. Como foi evidenciado anteriormente, este estudo detectou a presença de apenas uma criança infectada por Candida (0,9\%), dentre 114 neonatos críticos investigados, constatando uma taxa de incidência de 0,4/1.000 paciente/dia, sendo que o peso ao nascer deste recém-nascido foi $3.860 \mathrm{~g}$, diferentemente do encontrado na literatura acima citada.

A colonização por Candida tem sido mostrada em alguns estudos como um fator de risco independente para candidemia. Os sítios onde a colonização é mais freqüente incluem as mucosas da boca e intestino ${ }^{6}$ com a mucosa oral, variando de $20 \%$ a $55 \%{ }^{10}$ e mucosa do intestino $(74 \%)^{4}$. Nas crianças incluídas nesta investigação, a colonização na região perianal foi alta (68,9\%), sendo a Candida albicans a mais prevalente correspondendo a 51,6\% das amostras de mucosa perianal. Os fatores de risco relacionados significantemente com a colonização foram: idade gestacional entre 26 e 30 semanas e o uso prévio de antibiótico $(P \leq 0,05)$, entretanto o extremo baixo peso e o uso de intracath apresentaram Odds ratio igual a 3 e 7,13, respectivamente.

As fontes de candidíase invasiva são usualmente endógenas a partir da colonização das mucosas, principalmente intestinal, do neonato. 0 recém-nascido crítico é colonizado muito cedo, cerca de $10 \%$ destes se tornam colonizados na primeira semana de vida, e mais de 64\% estão colonizados após quatro semanas de hospitalização $0^{7}$ embora haja evidências de correlação entre colonização fúngica e doença invasiva em neonatos de extremo baixo peso ${ }^{1}$. Neste estudo não foi possível verificar se o neonato infectado estava previamente colonizado pelo fato de ter sido proveniente de outro hospital da região. Em relação ao grupo de pacientes colonizados, verificou-se que 19,3\% (22/114) dos neonatos estavam colonizados por Candida, identificadas como Candida albicans (50\%) e Candida não-albicans (50\%), sendo que o extremo baixo peso não apresentou frequiência estatisticamente significante, representando $13,7 \%$ dos casos.

Há evidências que as amostras Candida spp isoladas de pacientes de UTIN apresentam susceptibilidade diminuída aos agentes antifúngicos, devido ao aumento expressivo no seu uso ${ }^{13}$. Em relação ao grupo dos azóis, a Candida albicans apresenta resistência ao fluconazol < 5\%, enquanto a Candida krusei tem resistência intrínseca a este fármaco ${ }^{2}$. Neste trabalho, a maioria $(93,3 \%)$ das amostras de espécies de Candida foi resistente aos antifúngicos testados.

A taxa de mortalidade total por infecções fúngicas é alta, podendo atingir $50 \%$ em recém-nascidos ${ }^{11}$. No presente estudo, a mortalidade total foi de $11,8 \%$ naqueles neonatos que apresentaram sepse e 0 caso de candidemia não evoluiu para óbito.

Embora fosse detectado apenas um caso de candidemia durante o período investigado, aproximadamente $20 \%$ dos neonatos estavam colonizados por Candida, particularmente quando em uso de antibióticos, idade gestacional entre $26 \mathrm{e}$ 30 semanas e em uso de cateter vascular central umbilical. A colonização na mucosa intestinal foi mais expressiva do que na observada na boca $(68,9 \%$ vs $31,1 \%)$.

\section{REFERÊNCIAS}

1. Brencht M, Clerihew L, Mc Guire W. Prevention and treatment of invasive fungal infection in very low birthweight infants. Archives of Disease in Childhood: Fetal and Neonatal Edition 94:65-69, 2009.

2. Charlier C, Hart E, Lefort A, Ribaud P, Dromer F, Denning DW, Lortholary 0. Fluconazole for the management of invasive candidiasis: where do we stand after 15 years? The Journal of Antimicrobial Chemotherapy 57:384-410, 2006.

3. Colombo AL, Guimarães T. Epidemiologia das infecções hematogênicas por Candida spp. Revista da Sociedade Brasileira de Medicina Tropical 6:599-607, 2003

4. Gagneur A, Sizun J, Vernotle E, Parscau T, Quino DAM, Flohic LE, Baron R. Low rate of Candida parapsilosis - related colonization and infection in hospitalized preterm infants: a one-year prospective study. The Journal of Hospital Infection 48:193-197, 2001.

5. Garland JS, Uhing MR. Strategies to prevent bacterial and fungal infection in the Neonatal Intensive Care Unit. Clinics in Perinatology 36:1-13, 2009.

6. Gill SS, Swoboda SM, Johnson EA, Merz WG, Pelz RK, Lipsett PA, Hendrix CW. The association between anatomic site of Candida colonization invasive candidiasis, and mortality in critically ill surgical patients. Diagnostic Microbiology and Infectious Disease 55:293-301, 2006.

7. Kuzucu C, Durmaz R, Otlu B, Aktas E, Gulcan H, Cizmeci Z. Species distribution, antifungal susceptibility and clonal relatedness of Candida isolates from patients in neonatal and pediatric intensive care units at a medical Center in Turkey. The New Microbiologica 31:401-408, 2008

8. Linder N, Klinger G, Shalit I, Lewy I, Ashkenazi S, Haski G, Levit O, Sirota L. Treatment of candidaemia in premature infants: comparison of three amphotericin B preparations. The Journal of Antimicrobial Chemotherapy 52:663-667, 2003 
9. Lopez-Sastre JB, Gil D, Coto-Coballo MD, Fernandez B, Grupo de Hospitales Castrillo. Neonatal invasive candidiasis: a prospective multicenter study of 118 cases. American Journal of Perinatology 20:153-163, 2003.

10. Makhoul IR, Sujov P, Ardekian L, Kassis I, Smolkin T, Abu-Elnaa'j I, Tamir A, Laufer D. Factors influencing oral colonization in premature infants. The Israel Medical Association Journal 4:98-102, 2002.

11. Rao S, Ali U. Systemic fungal infections in neonates. Journal of Postgraduate Medicine, 51(supppl):27-29, 2005.

12. Richtmann R, Takagi NB, Valciloto E, Kusano E, Marques MRM, Baltieri SR, Arriero GD, Amaro ER. Candidemia em Unidade de Terapia Intensiva Neonatal: onde Estamos? Revista da Sociedade Paulista de Infectologia 1:5-9, 2005.

13. Roilides E, Farmaki E, Evdoridou J, Dotis J, Hatziionnidis E, Tsivitanidou M, Bibashi E, Filioti I, Sofianou D, Gil-Lamaignere C, Mueller FM, Kremenopoulos G. Neonatal candidiasis: analysis of epidemiology, drug susceptibility, and molecular typing of causative isolates. European Journal of Clinical Microbiology \& Infectious Diseases 23:745-750, 2004.

14. Saiman L, Ludington E, Dawson JD, Patterson JE, Rangel-Frausto S, Wiblin RT, Blumberg HM, Pfaller M, Rinaldi M, Edwards JE, Wenzel RP, Jarvis W. Risk factors for Candida species colonization of neonatal intensive care unit patients. The Pediatric Infectious Disease Journal 20:1119-1124, 2001.
15. Saiman L, Ludington E, Pfaller M, Rangel-Frausto S, Wiblin RT, Dawson J, Blumberg HM, Patterson JE, Rinaldi M, Edwards JE, Wenzel RP, Jarvis W. Risk factors for candidemia in neonatal intensive care unit patients. The Pediatric Infectious Disease Journal 19:319-324, 2000.

16. Sastre JBL, Cotallo GDC, Colomer BF, Castrillo GH. Neonatal Invasive Candidiasis: a prospective multicenter study of 118 cases. American Journal of Perinatology 20:153-163, 2003.

17. Stoll BJ, Hansen N, Fanaroff AA, Wright LL, Carlo WA, Ehrenkranz RA, Lemons JA, Donovan EF, Stark AR, Tyson JE, Oh W, Bauer CR, Korones SB, Shankaran S, Laptook AR, Stevenson DK, Papile LA, Poole WK. Late-onset sepsis in very-low birth weight neonates: the experience of the NICHD Neonatal Research Network. Pediatrics 110:285-291, 2002.

18. Vendettuoli V, Tana M, Tirone C, Posteraro B, La Sorda M, Fadda G, Romagnoli C, Vento G. The role of Candida surveillance cultures for identification of a preterm subpopulation at highest risk for invasive. The Pediatric Infectious Disease Journal, 27:114-116, 2008.

19. Vendettuoli V, Vento G, Tirone C, Posteraro B, Romagnoli C. Antifungal prophylaxis: identification of preterm neonates at highest risk for invasive fungal infection. Pediatrics 123: 368-369, 2009. 